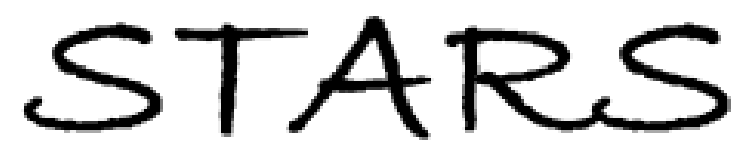

University of Central Florida

STARS

$1-1-2013$

\title{
Synchrotron X-ray measurement techniques for thermal barrier coated cylindrical samples under thermal gradients
}

\author{
Sanna F. Siddiqui \\ University of Central Florida \\ Kevin Knipe \\ University of Central Florida \\ Albert Manero \\ University of Central Florida \\ Carla Meid \\ Janine Wischek
}

Find similar works at: https://stars.library.ucf.edu/facultybib2010

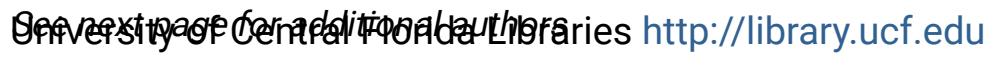

This Article is brought to you for free and open access by the Faculty Bibliography at STARS. It has been accepted for inclusion in Faculty Bibliography 2010 s by an authorized administrator of STARS. For more information, please contactSTARS@ucf.edu.

\section{Recommended Citation}

Siddiqui, Sanna F.; Knipe, Kevin; Manero, Albert; Meid, Carla; Wischek, Janine; Okasinski, John; Almer, Jonathan; Karlsson, Anette M.; Bartsch, Marion; and Raghavan, Seetha, "Synchrotron X-ray measurement techniques for thermal barrier coated cylindrical samples under thermal gradients" (2013). Faculty Bibliography 2010s. 2564.

https://stars.library.ucf.edu/facultybib2010/2564

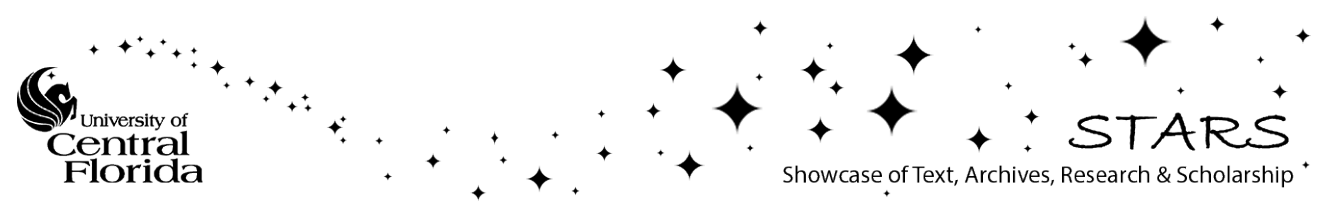




\section{Authors}

Sanna F. Siddiqui, Kevin Knipe, Albert Manero, Carla Meid, Janine Wischek, John Okasinski, Jonathan Almer, Anette M. Karlsson, Marion Bartsch, and Seetha Raghavan 


\section{Synchrotron X-ray measurement techniques for thermal barrier coated cylindrical samples under thermal gradients}

Sanna F. Siddiqui, Kevin Knipe, Albert Manero, Carla Meid, Janine Wischek, John Okasinski, Jonathan Almer, Anette M. Karlsson, Marion Bartsch, and Seetha Raghavan

Citation: Review of Scientific Instruments 84, 083904 (2013); doi: 10.1063/1.4817543

View online: https://doi.org/10.1063/1.4817543

View Table of Contents: http://aip.scitation.org/toc/rsi/84/8

Published by the American Institute of Physics

\section{Articles you may be interested in}

Role of mechanical loads in inducing in-cycle tensile stress in thermally grown oxide Applied Physics Letters 100, 111906 (2012); 10.1063/1.3692592

Stress and structural damage sensing piezospectroscopic coatings validated with digital image correlation AIP Advances 5, 037139 (2015); 10.1063/1.4916760

Tensile testing of materials at high temperatures above $1700^{\circ} \mathrm{C}$ with in situ synchrotron X-ray micro-tomography Review of Scientific Instruments 85, 083702 (2014); 10.1063/1.4892437

An Optical Fluorescence System for Quantitative Pressure Measurement in the Diamond-Anvil Cell Review of Scientific Instruments 44, 1 (1973); 10.1063/1.1685943

Strain and texture analysis of coatings using high-energy $x$-rays Journal of Applied Physics 94, 697 (2003); 10.1063/1.1582351

Nondestructive evaluation of the oxidation stresses through thermal barrier coatings using $\mathrm{Cr}^{3+}$ piezospectroscopy

Applied Physics Letters 69, 3754 (1996); 10.1063/1.117182

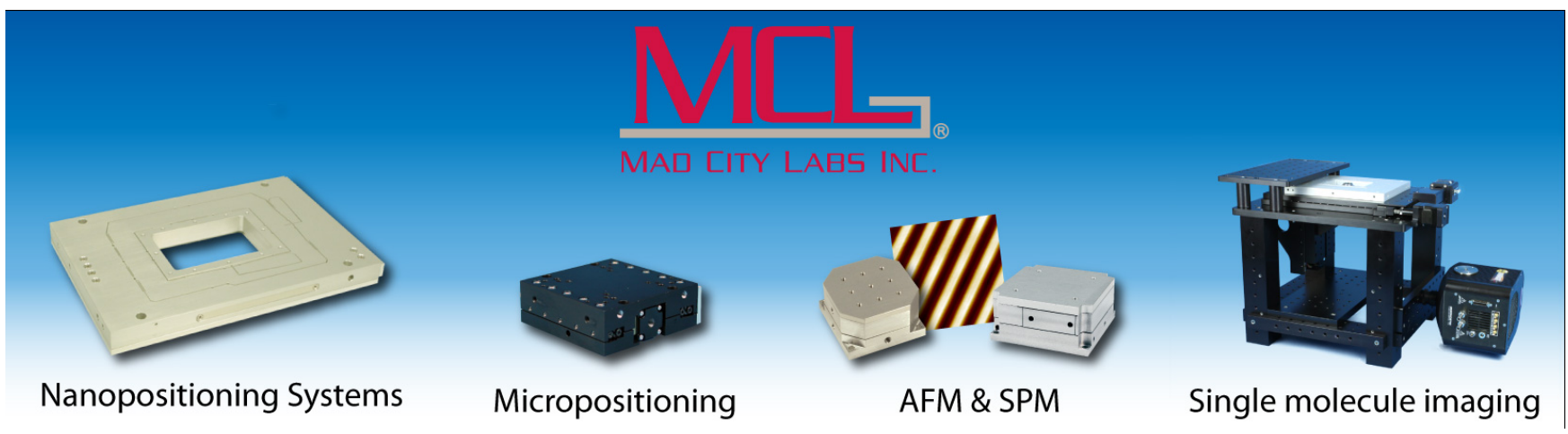




\title{
Synchrotron X-ray measurement techniques for thermal barrier coated cylindrical samples under thermal gradients
}

\author{
Sanna F. Siddiqui, ${ }^{1}$ Kevin Knipe, ${ }^{1}$ Albert Manero, ${ }^{1}$ Carla Meid, ${ }^{2}$ Janine Wischek, ${ }^{2}$ \\ John Okasinski, ${ }^{3}$ Jonathan Almer, ${ }^{3}$ Anette M. Karlsson, ${ }^{4}$ Marion Bartsch, ${ }^{2}$ \\ and Seetha Raghavan ${ }^{1, a)}$ \\ ${ }^{1}$ Department of Mechanical and Aerospace Engineering, University of Central Florida, Orlando, \\ Florida 32816, USA \\ ${ }^{2}$ German Aerospace Center (DLR), Institute of Materials Research, 51147 Cologne, Germany \\ ${ }^{3} X$-Ray Science Division, Advanced Photon Source, Argonne National Laboratory, Argonne. \\ Illinois 60439, USA \\ ${ }^{4}$ Cleveland State University, 2121 Euclid Avenue, Cleveland, Ohio 44115, USA
}

(Received 3 May 2013; accepted 22 July 2013; published online 12 August 2013)

\begin{abstract}
Measurement techniques to obtain accurate in situ synchrotron strain measurements of thermal barrier coating systems (TBCs) applied to hollow cylindrical specimens are presented in this work. The Electron Beam Physical Vapor Deposition coated specimens with internal cooling were designed to achieve realistic temperature gradients over the TBC coated material such as that occurring in the turbine blades of aeroengines. Effects of the circular cross section on the x-ray diffraction (XRD) measurements in the various layers, including the thermally grown oxide, are investigated using highenergy synchrotron x-rays. Multiple approaches for beam penetration including collection, tangential, and normal to the layers, along with variations in collection parameters are compared for their ability to attain high-resolution XRD data from the internal layers. This study displays the ability to monitor in situ, the response of the internal layers within the TBC, while implementing a thermal gradient across the thickness of the coated sample. The thermal setup maintained coating surface temperatures in the range of operating conditions, while monitoring the substrate cooling, for a controlled thermal gradient. Through variation in measurement location and beam parameters, sufficient intensities are obtained from the internal layers which can be used for depth resolved strain measurements. Results are used to establish the various techniques for obtaining XRD measurements through multi-layered coating systems and their outcomes will pave the way towards goals in achieving realistic in situ testing of these coatings. @ 2013 AIP Publishing LLC. [http://dx.doi.org/10.1063/1.4817543]
\end{abstract}

\section{INTRODUCTION}

Advances in achieving higher gas turbine inlet temperatures over recent decades have seen significant contributions from Thermal Barrier Coatings (TBC). These ceramic coatings have qualities of both low thermal conductivity required to impose a large temperature gradient between the combustion gases and the underlying superalloy, as well as the capability to withstand exposure to extreme temperatures. This facilitates both higher turbine inlet temperatures and a reduction in the load-bearing superalloy temperatures, providing higher thermal efficiencies and reduced thermal fatigue effects. Extensive research to understand the causes of failure modes and enhance the durability of these high temperature coatings has led to efforts in developing novel sample geometries and more realistic testing techniques to include effects of thermal gradients and/or mechanical loads.

Studies have been conducted to establish the relation between loading conditions and the presence of various failure modes such as delamination and spallation within the coatings. Previous results have shown specific failure mechanisms as a result of Thermal-Mechanical Fatigue (TMF) loading conditions. With high applied mechanical load, cracks are

a)Electronic mail: seetha.raghavan@ucf.edu seen to propagate perpendicularly from the bond coat/TGO interface, whereas low applied loads are dominated by delamination failure. ${ }^{1}$ Efforts to understand thermal gradient effects have led to methods such as torch heating of coated flat specimens and air cooling the back substrate surface, ${ }^{2,3}$ where ex situ studies reveal mud cracks due to high tensile stress on the surface. ${ }^{3}$ The use of $\mathrm{CO}_{2}$ lasers to induce the heat flux to the TBC surface opposite the air cooled substrate on flat specimens, enabled temperature measurements across the various layers of the TBC cross section with a gradient. ${ }^{4}$ An effective method for applying thermal gradients across the ceramic coating has been seen with the use of infrared lamps and internal cooling of tubular samples, and this has been used to study the damage evolution after cycling. ${ }^{5,6}$ Additional work has been done to study the effects of axial, radial, and circumferential thermal gradients. ${ }^{7}$ Combinations of both thermal gradients and mechanical loads have been applied through novel cylindrical sample geometries to establish that Thermal Gradient Mechanical Fatigue (TGMF) loading results in the development of cracks beneath the top coat and further the spallation process. ${ }^{8}$ Along with simulation studies, results have shown that a combination of the Thermally Grown Oxide (TGO) growth stress, applied tensile axial loading and creep relaxation of the TGO, leads to the initiation of these cracks under TGMF loading conditions. ${ }^{9}$ The difficulty 
associated with maintaining a thermal gradient in cylindrical samples that effectively simulates the operational conditions has been addressed previously for TGMF testing using quartz lamps and controlled coolant flow. ${ }^{6}$

Integral to the understanding of failure mechanisms in these layered systems is the strain evolution to failure under the different thermal and mechanical loading conditions. In conventional testing, information such as micro-structural properties and stress/strain distribution are obtained in an $e x$ situ approach between cycles or at various stages of the life span. The strain distribution at high temperature and its influence on crack initiation and propagation are largely unknown in terms of physical measurements. High spatial and strain resolution testing techniques such as Synchrotron XRay Diffraction (XRD) have capabilities for in situ measurements that can provide potentially significant findings. It is well known that high compressive stresses develop in the TGO during cooling from high temperature due to the thermal mismatch between the layers. ${ }^{10}$ Along with the advances in realistic testing techniques, in situ measurements present a unique opportunity to obtain an insight into the mechanics of the material response and capture the quantitative changes in strain under these conditions. High-energy $\mathrm{x}$-rays have been used to characterize strain in various ex situ studies of cycled TBC samples, providing important validation of quantitative strains from theoretical models. ${ }^{11}$ More unique in situ studies, on phase changes during oxide formation, have revealed new information on significant tensile stresses in the oxide accompanying the associated volume reduction. ${ }^{12}$ More recently, synchrotron XRD strain measurements on TBCs under thermal-mechanical loading conditions have shown the dependence on applied axial loads of the strain transition into the tensile region at high temperature in the TGO of as-coated specimens. ${ }^{13}$

The study presented here combines both the expertise in developing realistic testing conditions and in obtaining in situ synchrotron XRD measurements to achieve such an advanced testing capability for TBCs. The primary challenges lie in two areas; achieving high-resolution XRD measurements on the hollow cylindrical sample geometry and creating a controlled thermal gradient. The difficulty associated with obtaining XRD measurements on cylindrical samples for eventual stress and strain determination has led to the development of strain scanning techniques which use slits and sample rotation and/or translation to obtain depth resolved strains in bulk cylindrical samples. ${ }^{14}$ Additionally, work has been done on using XRD for determining the tri-axial stress state for cylindrical samples of cycled fatigued steel to resolve the stress components. ${ }^{15}$ The added complexity of the multilayered material system and the thermal gradient instrumentation with the furnace surrounding the sample is addressed in this work. Here, we show the ability to monitor, through XRD measurement techniques in both through thickness and grazing of the sample, the various internal layers in the coatings while applying a thermal gradient. This will show the capability to acquire measurements of distinguishable phases through the depth of each layer at multiple locations on the geometry. The approach to the second challenge of controlling the thermal gradients to achieve a high heat flux is presented through methods in focused radiation heating on the external surface in addition to cooling the substrate internally. Temperature monitoring of the system is used to establish techniques to control variable thermal gradients across the coating thickness. The outcomes unveil an advanced in situ test method for high temperature coatings research with extensive capabilities in monitoring time-correlated response of these materials under harsh and extreme environments. The potential for capturing otherwise unknown material behavior under operational environments is a much-needed contribution towards significant progress in the testing of these materials to achieve the next steps in durability enhancement.

\section{EXPERIMENTAL}

\section{A. Sample details}

The hollow cylindrical IN100 sample upon which XRD measurements were taken and thermal gradient control was achieved had an inner diameter of $4 \mathrm{~mm}$ and an outer diameter of $8 \mathrm{~mm}$. The EB-PVD TBC on the sample consisted of 7-8 wt. \% Yttria-Stabilized Zirconia (YSZ) ceramic topcoat with a thickness of $211 \pm 4 \mu \mathrm{m}$, an $\mathrm{Al}_{2} \mathrm{O}_{3}$ TGO layer thickness of approximately $0.3 \mu \mathrm{m}$, and a NiCoCrAlY metallic bond coat with a thickness of $118 \pm 4 \mu \mathrm{m}$. Sample preparation was conducted at the German Aerospace Center (DLR).

\section{B. XRD synchrotron measurements}

In situ synchrotron $\mathrm{x}$-ray diffraction measurements using 2 methods as seen in Figure 1 were taken at the 1-ID-C Beamline at the Advanced Photon Source in Argonne National Laboratory with a beam energy of $65 \mathrm{keV}$. In Method 1, the $\mathrm{x}$-ray beam grazes the edge of the sample, from which axial and radial components of strain can be measured within the layers of the thermal barrier coating. In Method 2, the $\mathrm{x}$-ray beam passes through the center of the sample, from which axial and circumferential strain components can be measured. Since these XRD measurements were taken with a 2D detector with a plane normal to the beam direction, multiple locations on the curvature were tested to demonstrate the ability to obtain the full strain tensor. The effectiveness of these two methods in obtaining strain measurements within the TBC layers was analyzed. In Method 1, 2D XRD measurements were taken tangentially by grazing the beam at various depths to attain diffraction rings for phases in the YSZ, TGO, and Bond Coat. In Method 2, the effect of varying beam size and exposure time on the intensity of a TGO peak was analyzed to determine the beam parameters that yielded the highest strain resolution measurement through the center of the sample. The schematic for the experimental setup is presented in Figure 2. To maintain access for beam diffraction, circular inlet and exit windows were created through the center of the front and back heater walls. High-energy synchrotron $\mathrm{x}$-rays allow the ability to use relatively small exit windows due to the small Bragg angle. The 1 in. diameter exit window shown in Figure 2 provided a $2 \theta$ diffraction angle of $8.5^{\circ}$. With the beam energy of $65 \mathrm{keV}$, full 


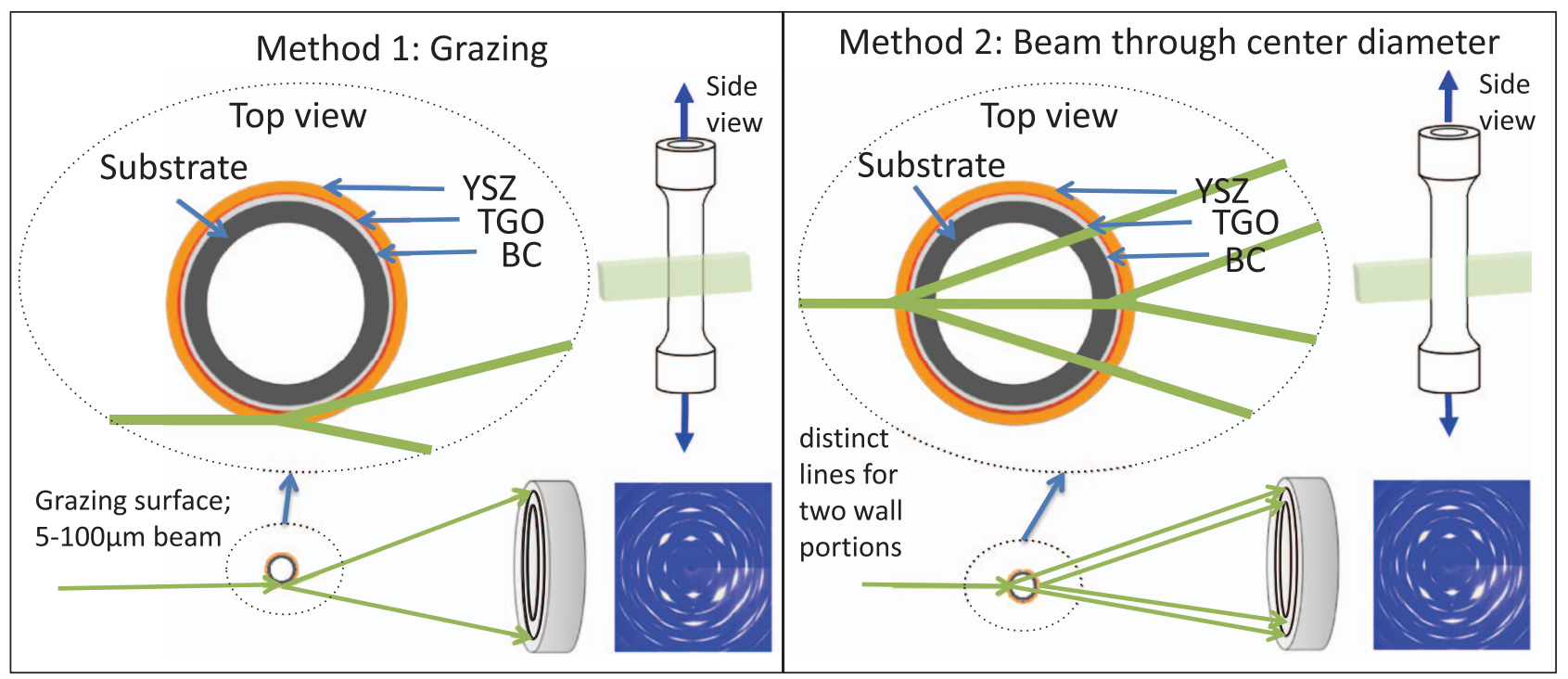

FIG. 1. Method 1: Grazing surface from 5 to $100 \mu \mathrm{m}$. 2D detector measures axial and radial components of stress. By moving the sample into the beam, information from different layers is collected. Method 2: Direct transmission along the center line. 2D detector measures wall-thickness-averaged axial and circumferential components.

diffraction rings can be measured to a d-spacing as small as $1.29 \AA$. The heater and sample were mounted in a configuration to allow four degrees of freedom for relative movement between the heater and sample. The heater was mounted and fixed to a location based on the stationary beam. The MTS load frame was grounded through stages providing three translational degrees of freedom and an axial rotation degree of freedom. To move the measurement location on the sample,

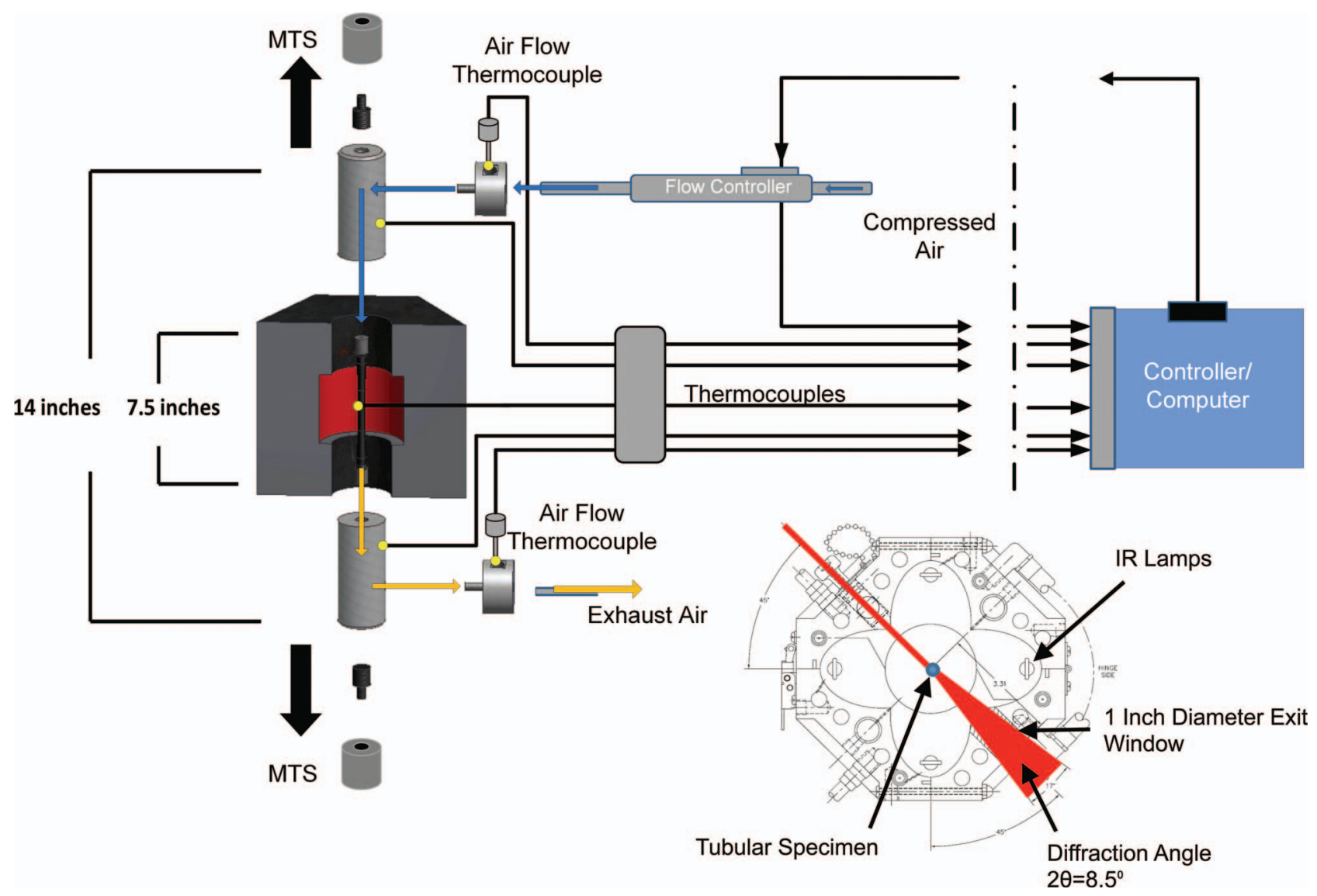

FIG. 2. Thermal gradient and mechanical load apparatus schematic: A system view of the thermal mechanical loading and data acquisition equipment. 
the load frame along with the sample were translated laterally relative to the beam.

\section{Control of thermal gradient}

The primary challenges associated with controlling the thermal gradient with a tubular sample are attaining the necessary thermal loading conditions for the desired gradient, as well as accurately monitoring the temperature conditions. Achieving this requires application of severe conditions to both the high and low temperature sides of the coating, while attempting to isolate both from the internal layers of the cross section to maintain a gradient across the coating thickness. As the stresses in each layer are dominated by cyclic thermal expansion mismatch, achieving the desired thermal gradient in both the thickness and surface plane directions is critical to strain measurements. To achieve this, cylindrical samples with external coatings are used for testing, which have certain benefits of uniformity and ability to achieve such gradients. Heating of a cylindrical surface allows the implementation of an enclosure heater minimizing heat loss as well as creating circumferentially consistent temperature values required for accurate representation of conditions. A focusing infrared heater with four lamps was used to create the necessary heat flux required to subject the coating to a large thermal gradient. This in-plane temperature consistency of cylindrical specimens is very beneficial to strain measurements as opposed to flat specimens which may present either heating of the exposed cross section or an in-plane gradient near the edges hindering strain results.

As illustrated in Figure 2, the thermal gradient was controlled using a flow controller which is capable of regulating the compressed air flow from 0 to 100 standard liters per minute (SLPM). This provided forced air cooling of the inner substrate wall of the tubular sample. A $0.35 \mathrm{~mm}$ diameter $\mathrm{S}$ type thermocouple with an exposed junction was used in a hoop configuration pulled around the specimen, providing a quick thermally responsive feedback for heater control. Inlet and exhaust air temperature data was collected by using two inline type $\mathrm{K}$ thermocouples giving measurements of total energy gains of the flow between the ports on both grips. Surface temperature measurements of the grips were taken just outside the heater, opposite the coolant port, and these served as indicators of total system steady-state as well as changes in heat loss across the grip material. The energy gain of the coolant flow along with the energy change across the sample and grip thicknesses were equated numerically to estimate the imposed temperature gradient across the coating thickness. To provide conditions realistic of the environment found surrounding a turbine blade, an infrared radiation chamber heater was employed. Radiation of four quartz lamps producing $2 \mathrm{~kW}$ each is focused to a center line with elliptical mirrors to provide optimal flux for a cylindrical specimen. This provides sufficient heating to sustain $1000{ }^{\circ} \mathrm{C}$ and above surface temperatures on the sample while maintaining the energy draws from cooling required to achieve large thermal gradients. The heater housing was sufficiently water cooled while the bulbs were air cooled to increase their longevity.

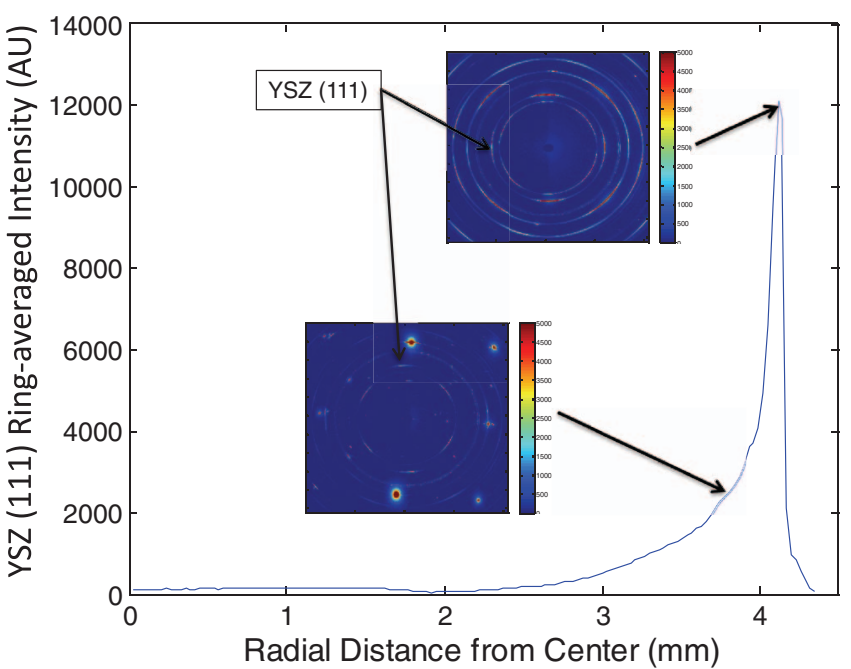

FIG. 3. Detected YSZ intensity from sample center to sample edge.

\section{RESULTS AND DISCUSSION}

\section{A. Effect of synchrotron XRD parameters on data}

In Method 1, in which the beam grazed the cylindrical sample, data was collected with a beam size of $(300 \times 30) \mu \mathrm{m}$ $\times \mu \mathrm{m}$ (height by width) and a frame count of 10 frames at one frame per second. To determine the geometry effects on the capability of distinguishing different phases, the XRD measurements were taken from the sample center to the YSZ outer radius using a step size of $30 \mu \mathrm{m}$ under zero loading conditions. At each measurement location, the diffraction intensity was averaged around the entire ring circumference producing averaged intensity vs. d-spacing. This average intensity gives insight into how the diffraction volume as well as the textured lattice plane reflection intensity varies when the $\mathrm{x}$-rays penetrate different locations on the geometry. Figure 3 shows the changes in intensity for the $t^{\prime}$ YSZ peak (111) through the entire scan. The YSZ scan displays a steady increase in measured intensity as the window approaches the YSZ inner radius of about $4.11 \mathrm{~mm}$. To display the phases at various depths, the XRD results are shown for Method 1 with grazing locations tangential through the YSZ and just inside the Bond Coat as well as Method 2 with penetrating directly through the center of the specimen. These locations are depicted in Figure 4 as A, B, and C. The averaged intensity vs. d-spacing
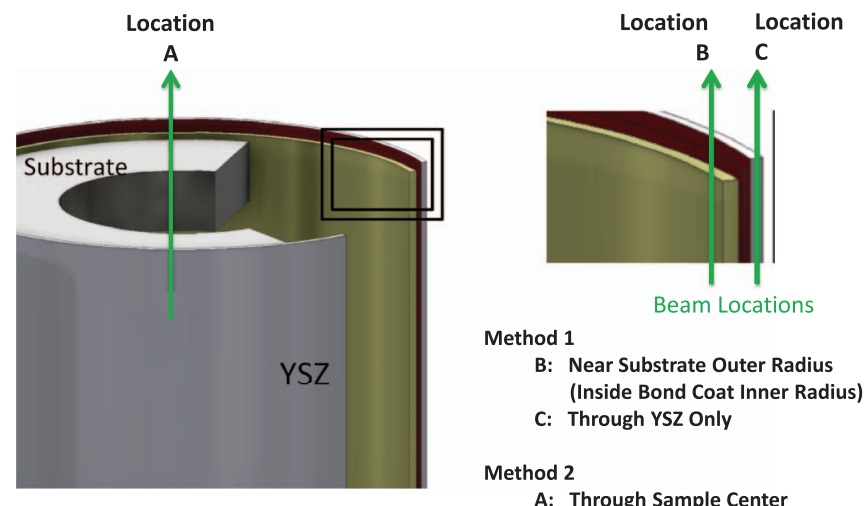

FIG. 4. XRD acquisition beam locations. 


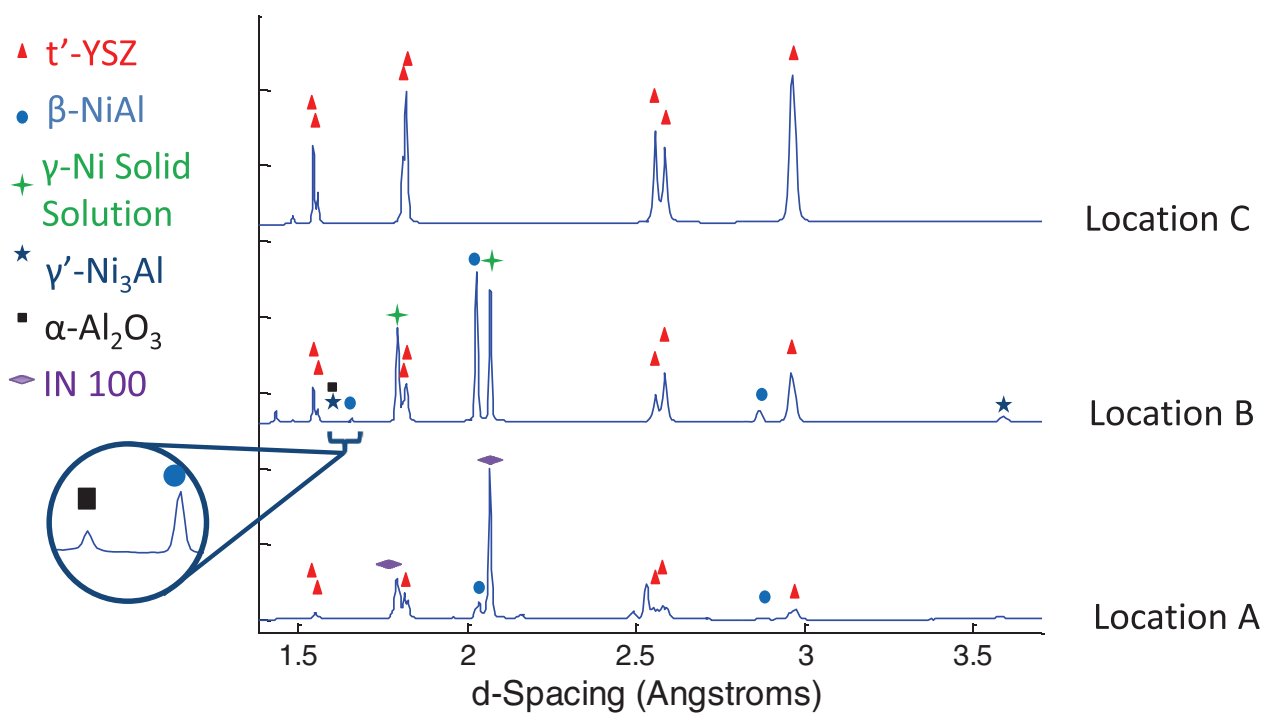

FIG. 5. XRD results for beam locations.

is compared at each of these locations with phase identification in Figure 5. At location $\mathrm{C}$, when shooting directly through the YSZ layer only, $t^{\prime}$ YSZ is the most prominent phase measured. At location B, the beam is grazing the substrate and penetrating through the Bond Coat, TGO, and YSZ. Here, the two phases which provide the highest intensities from the Bond Coat are $\beta-\mathrm{NiAl}$ phase and a solid solution $\gamma$-Ni phase. A $\gamma^{\prime}-\mathrm{Ni}_{3} \mathrm{Al}$ phase which displays an intermittent diffraction pattern representative of larger grain sizes is also developed. At this location, the largest distinguishable $\alpha-\mathrm{Al}_{2} \mathrm{O}_{3}$ peak is the (116) peak, which coincides with the $\gamma^{\prime}$ $\mathrm{Ni}_{3} \mathrm{Al}$ (210) peak. The smooth diffraction pattern $\alpha-\mathrm{Al}_{2} \mathrm{O}_{3}$ is distinguishable due to the intermittent diffraction pattern of the $\gamma^{\prime}-\mathrm{Ni}_{3} \mathrm{Al}$ phase, which appears visually as spots on the ring. To display the ability to analyze the TGO peak in terms of strain, the (116) peak is highlighted in Figure 5 at location $\mathrm{B}$. The presence of the well defined alumina peak with sufficient intensity, despite the minimal thickness of an ascoated specimen, provides the ability to fit the radial peak position precisely at points around the diffraction ring to determine two dimensional strains. The XRD results at location A highlight the need to test parameters such as window size and exposure time when measuring at the sample center. Precise measurement of the radial peak position is required and its deviation from a strain free radial position is then used for accurate strain calculations. The presence of false doublets due to double diffraction along with large, highly textured reflections from the Inconel 100 substrate presents complexities when attempting to analyze the 2D axial and circumferential strains at this location (Method 2).

The effect of varying XRD synchrotron parameters on the intensity of the TGO peak was analyzed due to the low diffraction volume of the TGO for the through center method, in order to obtain sufficient intensity for strain measurements. In Method 2, the X-ray beam was passed through the center diameter of the cylindrical sample to analyze the effects of varying beam size and exposure time on the intensity of TGO peak (116). At a constant exposure time of $0.5 \mathrm{~s}$ and frame count of 1 frame, the beam size was varied to $(25 \times 25)$,
$(50 \times 50),(100 \times 100),(200 \times 200),(300 \times 300),(400 \times 400)$, $(450 \times 450)$ and $(500 \times 500) \mu \mathrm{m} \times \mu \mathrm{m}$. In addition, at a constant beam size of $(40 \times 300) \mu \mathrm{m} \times \mu \mathrm{m}$, the exposure time was varied to $3,5,7,10,12$, and $15 \mathrm{~s}$. The effect of the window size and exposure time on the intensity of the alumina peak (116) is shown in Figures 6 and 7, respectively.

The relationship between the intensity of the TGO peak (116) and varying exposure time displayed a linear trend, with a correlation coefficient of $R^{2}=0.9983$, and a sufficient increase in intensity required for strain analysis. Figure 6 presents the effect of varying beam size in terms of beam area on the intensity of the TGO peak (116). This displays that the highest intensity of the TGO peak was obtained at a beam size of $(450 \times 450) \mu \mathrm{m} X \mu \mathrm{m}$, after which the curve is noticed to plateau. Additionally, one of the challenges in applying the through center method for strain measurements are the presence of doublet peaks acquired which result due to the beam passing through each coating layer twice as shown in Figure 1. The presence of these doublet peaks requires additional analysis techniques in order to fit a strain curve.

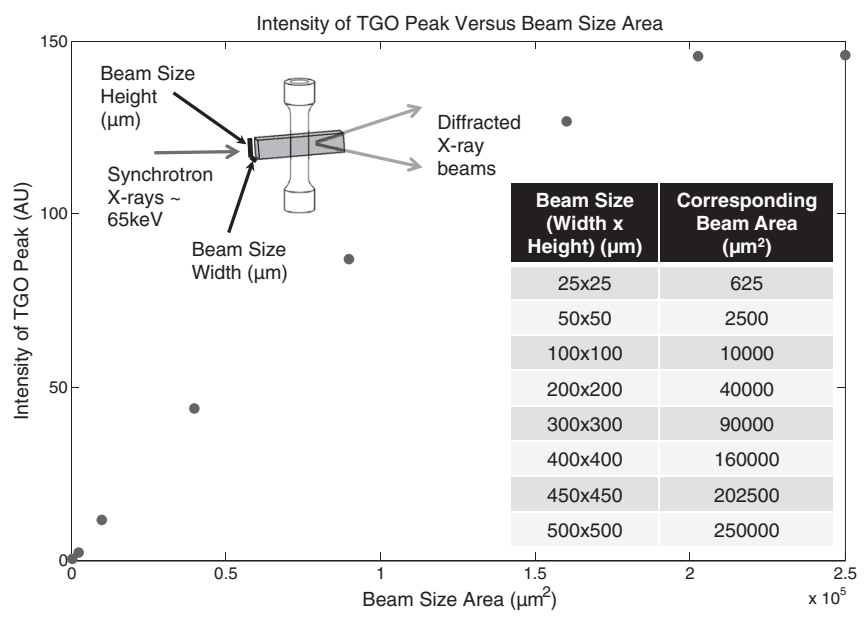

FIG. 6. Detected TGO intensity with varying beam size area, beam location A. 


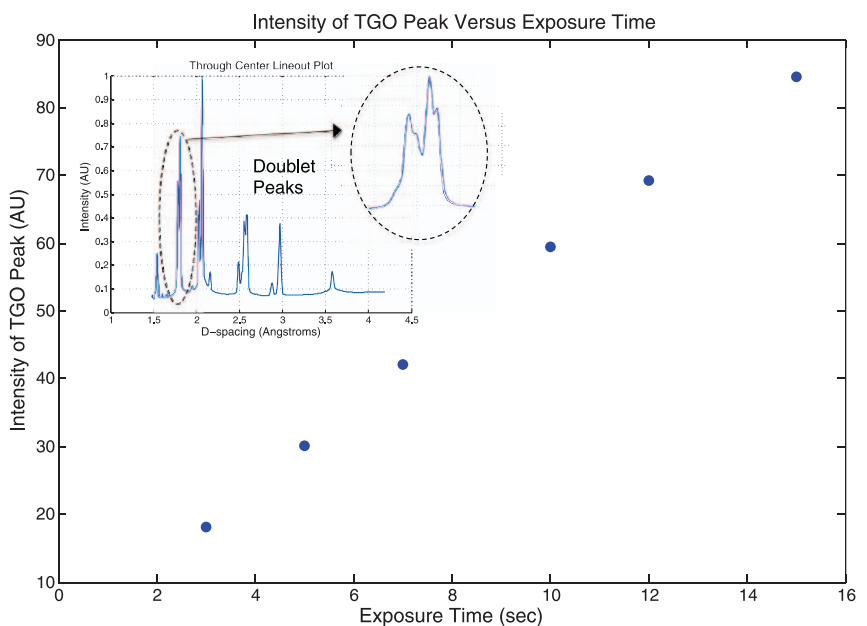

FIG. 7. Detected TGO intensity with varying exposure time, beam location A.

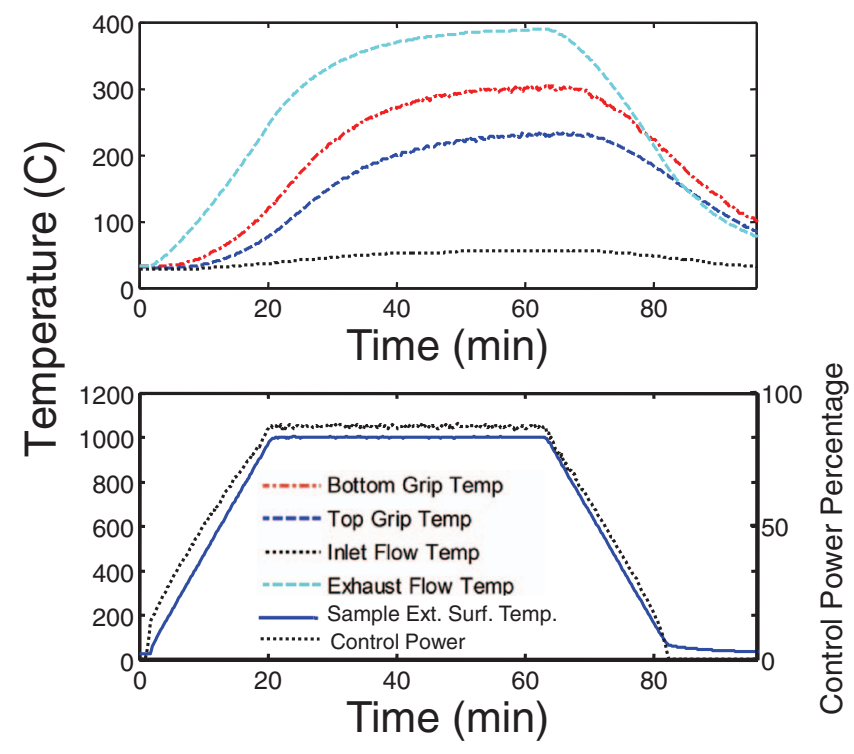

FIG. 8. Thermal measurement for a cycle with internal cooling.
However, the use of the through center method is effective in yielding sufficient intensities to obtain axial and circumferential components of strain. From the earlier described grazing results, clear peak position acquisition for various peaks of each layer provide strain components in both axial and radial directions. These results show the ability of both these methods, with the appropriate parameters, to measure multiple locations on the curvature in order to yield the full strain tensor required for in situ strain determination.

\section{B. Results from control of thermal gradient}

Figure 8 provides the temperature measurements of a thermal cycle. The sample surface was heated up at a rate of $50{ }^{\circ} \mathrm{C}$ per minute from ambient to $1000{ }^{\circ} \mathrm{C}$ and held at high temperature for $40 \mathrm{~min}$ after which it was ramped back down to ambient temperature. The flow rate was held constant at 75 SLPM throughout the cycle. It is notable that there is a lag between the bottom (exhaust) grip temperature and the exhaust flow temperature. There is also a slight rise in inlet air temperature readings, as the housing around the probe has some heat conducted to it.

To test the temperature response to variation in flow-rate, a ramp increase in coolant flow rate was applied to the system at high temperature. The entire system was allowed time to reach steady-state with an external sample surface temperature of $1000{ }^{\circ} \mathrm{C}$. The internal airflow was then increased linearly at a rate of 0.25 SLPM per minute from 0 to 75 SLPM. This rate was chosen in an attempt to mitigate transient behavior of the gradient across the midsection thickness of the coated specimen while minimizing the time for increased heat loss of the sample grips. The effects of this can be observed in Figure 9 where an increase in the flow rate produces a smooth curve in the exit coolant temperature but near constant grip temperatures during the variation period. The exit coolant temperature reaches a maximum of $395{ }^{\circ} \mathrm{C}$ at 60 SLPM with the inlet temperature taking longer to tend towards steady state. This reflects the ability of the inlet ambient air temperature to overcome the unwanted heating effect of the grip on this thermocouple. The surface grip

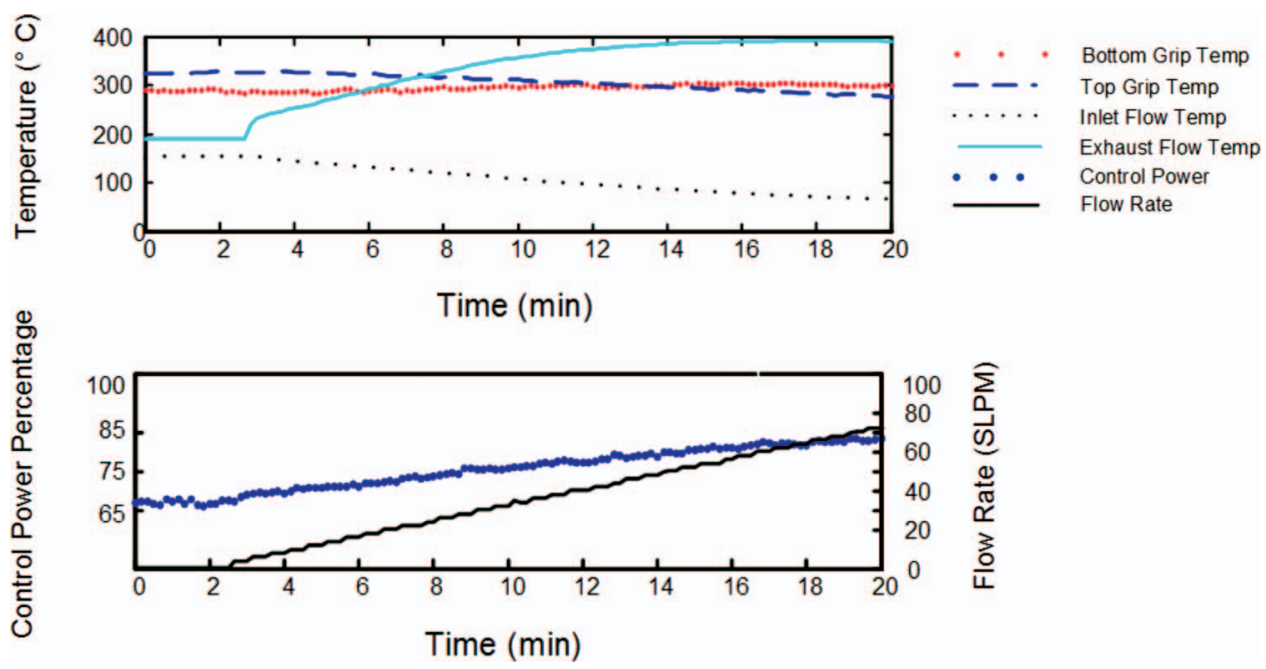

FIG. 9. Thermal measurements for a cycle with a linear increase in flow rate. 
temperatures show relatively small changes giving the desired limiting of the energy variations in the grip material.

These measurements display the ability to apply a large enough heat flux to maintain surface temperatures in the range of realistic operating conditions while sustaining large energy draws at the inner substrate. While applying coolant levels corresponding to the largest energy draws, surface temperatures were maintained at and above $1000{ }^{\circ} \mathrm{C}$, displaying sufficient heating at maximum thermal gradient. The temperature trends shown in these studies present, qualitatively, the ability to create large thermal gradients through the measured energy drawn from the system by the coolant temperature increase.

\section{CONCLUSION}

The tested thermal loads of this experiment were successful in showing the ability to control thermal gradients applicable to turbine operating conditions. The IR heater maintained temperatures on the ceramic surface in excess of $1000{ }^{\circ} \mathrm{C}$ while implementing large cooling effects across the substrate. This thermal gradient setup was tested and successfully maintained an external temperature of $1000{ }^{\circ} \mathrm{C}$ throughout the application of 0-75 SLPM internal air coolant. This study tested the ability to obtain depth resolved XRD measurements of each internal TBC layer at multiple locations on the curvature. This included grazing the surface of the sample and passing the $\mathrm{x}$-ray beam through the center of the sample. From grazing of the sample, phases in the YSZ, Bond Coat, and TGO were clearly distinguishable throughout the depth of the coating. From through center measurements on TGO peak (116), it was determined that the maximum intensity occurred at a beam size of $(450 \times 450) \mu \mathrm{ms}$. A linear trend was noted between intensity of the TGO peak and varying exposure time. The presence of doublet peaks from the through center measurement method is an added complexity in the determination of quantitative strain from these measurements. Through variable testing of location and beam parameters, it is displayed that both through center and grazing techniques are viable and can be used in combination to derive the full strain tensor in each internal layer.

\section{ACKNOWLEDGMENTS}

This material is based upon work supported by the National Science Foundation grants (Grant Nos. OISE 1157619 and CMMI 1125696) and by the German Science Foundation (DFG) grant (Grant No. SFB-TRR103), Project A3. This material is additionally based upon work supported by the National Science Foundation Graduate Research Fellowship Program under Grant No. 1144246 awarded to Sanna F. Siddiqui. Use of the Advanced Photon Source, an Office of Science User Facility operated for the U.S. Department of Energy (DOE) Office of Science by Argonne National Laboratory, was supported by the U.S. DOE under Contract No. DE-AC02-06CH11357.

${ }^{1}$ E. Tzimas, H. Mullenjans, S. D. Peteves, J. Bressers, and W. Stamm, "Failure of thermal barrier coating systems under cyclic thermomechanical loading," Acta Mater. 48, 4699-4707 (2000).

${ }^{2}$ J. M. Drexler, A. Aygun, D. Li, R. Vaben, T. Steinke, and N. P. Padture, "Thermal-gradient testing of thermal barrier coatings under simultaneous attack by molten glassy deposits and its mitigation," Surf. Coat. Technol. 204, 2683-2688 (2010).

${ }^{3}$ A. M. Limarga, R. Va $\beta$ en, and D. R. Clarke, "Stress distributions in plasmasprayed thermal barrier coatings under thermal cycling in a temperature gradient," J. Appl. Mech. 78, 011003 (2011).

${ }^{4}$ M. M. Gentleman, V. Lughi, J. A. Nychka, and D. R. Clarke, "Noncontact methods for measuring thermal barrier coating temperatures," Int. J. Appl. Ceram. Technol. 3, 105-112 (2006).

${ }^{5}$ H. Aleksanoglu, A. Scholz, M. Oechsner, C. Berger, M. Rudolphi, M. Schütze, and W. Stamm, "Determining a critical strain for APS thermal barrier coatings under service relevant loading conditions," Int. J. Fatigue 53, 40-48 (2013).

${ }^{6} \mathrm{M}$. Bartsch, G. Marci, K. Mull, and C. Sick, "Fatigue testing of ceramic thermal barrier coatings for gas turbine blades," Adv. Eng. Mater., 1(2), 127-129 (1999)

${ }^{7}$ V. Maurel, A. Koster, and L. Remy, "An analysis of thermal gradient impact in thermal mechanical fatigue testing," Fatigue Fract. Eng. Mater. Struct. 33, 473-489 (2010).

${ }^{8}$ M. Bartsch, B. Baufeld, S. Dalkilic, L. Chernova, and M. Heinzelmann, "Fatigue cracks in a thermal barrier coating system on a superalloy in multiaxial thermomechanical testing," Int. J. Fatigue 30, 211-218 (2008).

${ }^{9}$ M. T. Hernandez, A. M. Karlsson, and M. Bartsch, "On TGO creep and the initiation of a class of fatigue cracks in thermal barrier coatings," Surf. Coat. Technol. 203, 3549-3558 (2009).

${ }^{10}$ A. G. Evans, D. R. Mumm, J. W. Hutchinson, G. H. Meier, and F. S. Pettit, "Mechanisms controlling the durability of thermal barrier coatings," Prog. Mater. Sci. 46, 505-553 (2001).

${ }^{11}$ J. Thornton, S. Slater, and J. Almer, "The measurement of residual strains within thermal barrier coatings using high-energy x-ray diffraction," J. Am. Ceram. Soc. 88, 2817-2825 (2005).

${ }^{12}$ B. W. Veal, A. P. Paulikas, and P. Y. Hou, "Tensile stress and creep in thermally grown oxide," Nature Mater. 5, 349-351 (2006).

${ }^{13}$ R. Diaz, M. Jansz, M. Mossaddad, S. Raghavan, J. Okasinski, J. Almer, H. Pelaez-Perez, and P. Imbrie, "Role of mechanical loads in inducing in-cycle tensile stress in thermally grown oxide," Appl. Phys. Lett. 100, 111906 (2012).

${ }^{14}$ U. Lienert, J. Almer, D. Haeffner, Y. Gao, and W. Carter, "Nondestructive strain tensor scanning within samples of cylindrical symmetry," in American Institute of Physics Conference Series, (May 2004), Vol. 705, pp. 1074-1077.

${ }^{15} \mathrm{P}$. S. Prevéy and P. W. Mason, "The use of x-ray diffraction to determine the triaxial stress state in cylindrical specimens," Practical Applications of Residual Stress Technology (American Society for Metals, 1991), pp. $77-81$. 